\title{
Weights on Polytopes
}

\author{
P. McMullen \\ Department of Mathematics, University College London, \\ Gower Street, London WC11E 6BT, England \\ p.momullen@ucl.ac.uk
}

\begin{abstract}
The g-theorem describes the possible face-vectors of a simple polytope $P$. Much of the author's proof of the necessity of its conditions, while working within the polytope algebra $\Pi$, in fact only used the spaces of weights on $P$. Even though this proof was conceptually easier than the original, which employed techniques from algebraic geometry, nevertheless the properties of $\Pi$ which are needed still require some effort to establish, despite a recent simpler approach to $\Pi$ itself. In the earlier paper, doubt was expressed about whether two basic results could be proved directly for weights; later, it appeared that there might also be a possible problem concerning an alternative definition of the product of certain weights. In this paper these questions are settled, in the context of developing an independent theory of an algebra $\Omega$ of weights on polytopes. Since the construction of $\Omega$ is more approachable than that of $\Pi$, a yet easier proof of the $g$-theorem is now available.
\end{abstract}

\section{Introduction}

In [12] we showed how the necessity of the conditions of the $g$-theorem concerning the possible $f$-vectors of simple $d$-polytopes, which was conjectured in [10], and proved in [16] using deep techniques of algebraic geometry, could be established using the polytope algebra $\Pi$ of [11]. (The sufficiency of the conditions was proved in [2].) Thus a proof is now available, which is "elementary", in the sense that it works entirely within convexity. (The polytope algebra itself is not exactly straightforward, although a shorter approach to part of the theory has been expounded in [14].)

However, it has been pointed out to us by Gil Kalai (private communication) that there is a potential lacuna in the proof. More specifically, in Section 5 of [12] a special case of the multiplication in $\Pi$ is extended to what are called weights, which are only subsequently shown actually to belong to $\Pi$ all along. The question raised was whether it was clear that the induced multiplication of a weight by a general element of $\Pi$ was well defined. The same flaw could also vitiate the proof of the main result of [13] (and its subsequent applications), which assumes this fact as well. 
In this paper we bridge the gap. In fact, we show considerably more; a statement we made in the first part of Section 5 of [12] is also incorrect, but fortunately in a beneficial sense. It turns out that, contrary to what we suggested there, it is relatively easy to show that the product of two weights (as an extension of the product in $\Pi$ ) is well defined, and is also a weight (see Section 6). The basis of this result is something rather deeper; in Section 5 we show that linear mappings on polytopes induce homomorphisms on weight spaces. A consequence is that the weights form an algebra $\Omega$; we develop various properties of this algebra here.

It is worth noticing that the multiplication on weights just extends a geometric method of calculating mixed volumes (compare [1] and, for a more abstract approach, [11]). Indeed, it might well be appropriate to think of the weight algebra $\Omega$ as the algebra of mixed volumes, since we prove in Section 11 that $\Omega$ is generated as an abelian group by mixed volumes (or even actual volumes) of faces of polytopes.

A further simplification of [12] now results, in that we may throw away the scaffolding of the polytope algebra, and carry out the whole proof without it. In fact, the essential parts of the proof in [12], as applied to the g-theorem, did only employ weights. The reader who may wish to work through [12] in the light of the weight algebra can effectively discard much of that paper. In the final section we detail the appropriate modifications and substitutions to [12]; we also take the opportunity to provide some corrections to it. (We thank Gil Kalai and Isabella Sheftel for diligently working through [12], and raising searching questions about these various points. It is at Gil Kalai's prompting that we have added this section.)

An alternative construction of the weight algebra is described in [4]. However, there are considerable differences between that approach and ours. The starting point of [4] is the Chow ring; in other words, the much heavier machinery of algebraic topology provides the background. It should be noticed that this context restricts the scalars involved to be rational. In contrast, and in keeping with our general view that machinery should be kept as simple as possible, here we use only fairly low-level technology. While we do not carry out our study in such generality, it may also be remarked that slight changes in language will enable our constructions to be performed in vector spaces over arbitrary ordered fields, which need not be archimedean.

We should further draw attention to connexions between weights and the concepts of rigidity and stress on polytopes; for representative samples of the literature see [6]-[8] and [17]. We hope to explore these relationships more deeply in a subsequent paper.

\section{Minkowski Linear Combinations}

We frequently use Minkowski linear combinations in this paper, and so we discuss the basic notions here. We largely follow Section 14 of [5] to which we refer for further details, but we slightly vary some terminology, and also introduce notation which is more convenient for our purposes.

We denote by $\mathcal{P}$ the family of all (convex) polytopes in $\mathbb{E}^{d}$, by $\mathcal{F}(P)$ the set of all faces of $P \in \mathcal{P}$, and by $\mathcal{F}^{r}(P)$ the subset of its $r$-faces ( $r$-dimensional faces). The Minkowski or vector sum $P+Q$ of $P, Q \in \mathcal{P}$ is given by

$$
P+Q:=\{x+y \mid x \in P, y \in Q\}
$$


We often abbreviate $\{t\}$ to $t$, particularly in the context of a translate $P+t$, with $P \in \mathcal{P}$ and $t \in \mathbb{E}^{d}$. If $P, Q \in \mathcal{P}$ are such that $P=Q+Q^{\prime}$ for some $Q^{\prime} \in \mathcal{P}$, then we call $Q$ a summand of $P$. Note that a translate of a summand is also a summand. The scalar multiple of $\lambda \in \mathbb{R}$ is

$$
\lambda P:=\{\lambda x \mid x \in P\}
$$

almost invariably we have $\lambda \geq 0$. As usual, we write $P-Q$ for $P+(-1) Q$, and so on. We write $Q \preceq P$ if $\lambda Q$ is a summand of $P$ for some $\lambda>0$; observe that this relation is clearly transitive. Further, we write $P \simeq Q$ for the equivalence relation given by $P \preceq Q \preceq P$.

We define the support functional $\eta(P, \cdot)$ of $P \in \mathcal{P}$ by

$$
\eta(P, u):=\max \{\langle x, u\rangle \mid x \in P\}
$$

for $u \in \mathbb{E}^{d}$. If $u$ is a unit vector, then $\eta(P, u)$ is the support parameter of $P$ in direction $u$. For $u \in \mathbb{E}^{d}$, we denote by

$$
P_{u}:=\{x \in P \mid\langle x, u\rangle=\eta(P, u)\}
$$

the face of $P$ in direction $u$, and if $u \neq o$, the zero vector in $\mathbb{E}^{d}$, then

$$
H(P, u):=\left\{x \in \mathbb{E}^{d} \mid\langle x, u\rangle=\eta(P, u)\right\}
$$

is the corresponding support hyperplane with outer normal $u$. Note that $\eta(t, \cdot)=\langle t, \cdot\rangle$ for $t \in \mathbb{E}^{d}$, and that $P_{o}=P$.

We also denote by $u_{F, P}$ the outer unit normal vector to a polytope $P$ at a facet $F$ (face of codimension 1). It will be a convention that such a vector is always taken to be parallel to $P$ when $\operatorname{dim} P<d$.

It is immediate that, for Minkowski sums and scalar multiples, we have

$$
\begin{aligned}
\eta(P+Q, \cdot) & =\eta(P, \cdot)+\eta(Q, \cdot), \\
\eta(\lambda P, \cdot) & =\lambda \eta(P, \cdot),
\end{aligned}
$$

when $P, Q \in \mathcal{P}$ and $\lambda \geq 0$. It thus follows that, for such $P, Q$, and $\lambda$,

$$
\begin{aligned}
(P+Q)_{u} & =P_{u}+Q_{u}, \\
(\lambda P)_{u} & =\lambda P_{u},
\end{aligned}
$$

for each vector $u$. Hence, if $Q \preceq P$, then $Q_{u} \preceq P_{u}$ for each normal vector $u$.

The normal cone $N(F, P)$ to $P \in \mathcal{P}$ at a (non-empty) face $F$ is the cone of outer normals to support hyperplanes of $P$ which contain $F$; that is,

$$
N(F, P):=\left\{u \in \mathbb{E}^{d} \mid F \subseteq P_{u}\right\} .
$$

The normal fan of $P$ is then

$$
\mathcal{N}(P):=\{N(F, P) \mid F \in \mathcal{F}(P)\},
$$

the complex consisting of the normal cones to the faces of $P$. 
A necessary and sufficient condition for $Q \preceq P$ is that $\mathcal{N}(P)$ be a refinement of $\mathcal{N}(Q)$; indeed, for a Minkowski sum, $\mathcal{N}(P+Q)$ is the common refinement of $\mathcal{N}(P)$ and $\mathcal{N}(Q)$. Thus if $P \simeq Q$, then $\mathcal{N}(P)=\mathcal{N}(Q)$, so that $P$ and $Q$ are strongly isomorphic; that is, the polytopes are isomorphic, and the isomorphism between their faces is given by $P_{u} \leftrightarrow Q_{u}$ for each vector $u$. We denote by $\mathcal{K}(P)$ the strong isomorphism class of $P \in \mathcal{P}$; that is,

$$
\mathcal{K}(P):=\{Q \in \mathcal{P} \mid Q \simeq P\} .
$$

Looking at strong isomorphism from the viewpoint of normal cones accounts for the alternative term normally equivalent sometimes used instead of strongly isomorphic; see for instance [4].

We may also observe here that if $Q$ is any polytope, and $P$ is obtained from $Q$ by any sufficiently small parallel displacements of its facet hyperplanes, then $\mathcal{N}(P)$ refines $\mathcal{N}(Q)$, so that $Q \preceq P$. In particular, there is always a simple polytope $P$ with the same number of facets as $Q$, such that $Q \preceq P$; a simple $d$-polytope $P$ has precisely $d$ facets through each of its vertices. A useful remark for the future is the following.

Lemma 2.1. If $Q$ is any polytope in $\mathbb{E}^{d}$, then a simple d-polytope $P$ exists such that $Q \preceq P$.

Proof. By adding any $d$-polytope to $Q$ if necessary, we may assume that $\operatorname{dim} Q=d$. If $P$ is obtained from $Q$ by any sufficiently small parallel displacements of its facet hyperplanes, then $\mathcal{N}(P)$ refines $\mathcal{N}(Q)$, so that $Q \preceq P$, as required.

\section{Weights}

We begin with a basic definition. An $r$-weight on $P \in \mathcal{P}$ is a real-valued function $a$ on $\mathcal{F}^{r}(P)$ which satisfies the Minkowski relation on each $G \in \mathcal{F}^{r+1}(P)$, namely

$$
\sum_{F \in \mathcal{F}^{r}(G)} a(F) u_{F, G}=o,
$$

where the normal vector $u_{F . G}$ is defined as in Section 2. Note that this condition is satisfied when $a(F)=\operatorname{vol}_{r}(F)$ is the ordinary $r$-volume of $F$; of course, this motivates the definition. Throughout, we adopt the convention for an $r$-weight $a$ that $a(F)=0$ if $F$ is a face of $P$ with $\operatorname{dim} F \neq r$. We denote by $\Omega_{r}(P)$ the real vector space of $r$-weights on $P$, and write $\Omega(P):=\bigoplus_{r=0}^{d} \Omega_{r}(P)$.

Before we proceed further, we look at some particular values of $r$. If $r=0$, then a weight takes the same value on each vertex (the Minkowski relation implies that this is true for the two vertices of an edge). If $r=d-1$, and $P$ is a $d$-polytope with $n$ facets, then $\Omega_{d-1}(P)$ has dimension $n-d$. To see this, choose a linear basis of $\mathbb{E}^{d}$ from among the outer normal vectors to the facets of $P$, and then observe that weights $a(F)$ can be assigned arbitrarily to those facets $F$ of $P$ whose normals do not belong to this basis. Finally, again when $P$ is a $d$-polytope, $\Omega_{d}(P) \cong \mathbb{R}$, since $P$ is the only $d$-face of itself (the Minkowski relations now have no force). 
In fact, what we have here is not as general as it could be. There is an abstract concept of weight; we repeat some basic definitions from [11] (compare also [4]). Let $L$ be an $s$-dimensional linear subspace of $\mathbb{E}^{d}$, and denote by $\mathcal{C}(L)$ the family of polyhedral cones in $L$ with apex $o$. Then the cone group $\hat{\Sigma}(L)$ is the abelian group with a generator $\langle K\rangle$ for each $K \in \mathcal{C}(L)$; these generators satisfy the relations (V): $\left\langle K \cup K^{\prime}\right\rangle+\left\langle K \cap K^{\prime}\right\rangle=$ $\langle K\rangle+\left\langle K^{\prime}\right\rangle$ whenever $K \cup K^{\prime} \in \mathcal{C}(L)$ also, and $(S):\langle K\rangle=0$ if $\operatorname{dim} K<s$; thus $\hat{\Sigma}(L)$ is the abstract group for simple valuations on $\mathcal{C}(L)$. Of course, $\hat{\Sigma}(L)$ has a rather trivial group structure, and we can identify one of its elements as a finite family of cones with integer multiplicities (which may be negative).

For $s=0, \ldots, d$, the cone group $\hat{\Sigma}^{s}$ is defined to be

$$
\hat{\Sigma}^{s}:=\bigoplus_{\operatorname{dim} L=s} \hat{\Sigma}(L)
$$

Last, the full cone group $\hat{\Sigma}$ is

$$
\hat{\Sigma}:=\bigoplus_{s=0}^{d} \hat{\Sigma}^{s} .
$$

Now let $a \in \mathbb{R} \otimes \hat{\Sigma}^{d-r}$, say $a=\sum_{K} \alpha_{K} \otimes\langle K\rangle$. The component cones $K$ together have finitely many edges, in the directions of the unit vectors $u_{1}, \ldots, u_{n}$, say. Adjoining suitable extra $u_{j}$ if necessary (which would refine the cones $K$ ), we may suppose that the $u_{j} \operatorname{span} \mathbb{E}^{d}$ positively. There are finitely many strong isomorphism classes of $d$-polytopes whose facets have the $u_{j}$ as normal vectors to their facets; let $P$ be the Minkowski sum of one representative from each of these classes. The normal cones to the $r$-faces of $P$ may further refine the cones $K$. If $F \in \mathcal{F}^{\prime}(P)$, then extend the definition of $a$ by setting

$$
a(F):=\sum_{N(F, P) \subseteq K} \alpha_{K}
$$

Then we call $a$ an $r$-weight if $a \in \Omega_{r}(P)$, so that it is an $r$-weight on some polytope in the previously understood sense. We denote by $\Omega_{r}$ the vector space of all $r$-weights in $\mathbb{E}^{d}$, and write $\Omega:=\bigoplus_{r=0}^{d} \Omega_{r}$.

It remains to remark that we can verify the Minkowski relations directly from the cones, so that the definition just given does not really depend on the particular polytope $P$ on which they are checked; we repeat the appropriate part of [11]. A $k$-frame is an ordered orthogonal set $U=\left(u_{1}, \ldots, u_{k}\right)$ of unit vectors in $\mathbb{E}^{d}$. We extend the notation of Section 2 by defining recursively the face of a polytope $P$ in direction $U$ to be

$$
\left.P_{U}:=\left(P_{\left(u_{1}, \ldots, u_{k-1}\right)}\right)\right)_{u_{k}},
$$

with $P_{\emptyset}:=P$. Then $P_{U}$ is a face of $P$ of dimension at most $d-k$.

Let $K$ be a $k$-cone and let $U=\left(u_{1}, \ldots, u_{k}\right)$ be a $k$-frame. We say that $U$ is adapted to $K$ if there is a sequence $\{o\}=Q_{0} \subseteq Q_{1} \subseteq \cdots \subseteq Q_{k}=K$ of faces of $K$, such that $u_{j} \in Q_{j}-Q_{j-1}$ for each $j=1, \ldots, k$. Geometrically, this means that $u_{j}$ lies in the cone generated by $Q_{j}$ with the apex a relatively interior point of $Q_{j-1}$. If $F$ is an $r$-face of the polytope $P$, and $U$ is a $(d-r)$-frame, then $P_{U}=F$ precisely when $U$ is 
adapted to the normal cone $N(F, P)$. Following [11], but with a different notation, if $a=\sum_{K} \alpha_{K} \otimes\langle K\rangle$ (in the sense above) is an $r$-weight, we define $a_{U}$ by

$$
a_{U}:=\sum_{U \text { adapted to } K} \alpha_{K}
$$

The abstract criterion for an $r$-weight $a$ is thus

$$
\sum_{u \perp W} a_{(W, u)} u=o
$$

for each $(d-r-1)$-frame $W$. When $a \in \Omega_{r}(P)$ for some polytope $P$, this coincides with the previous definition, in view of our convention that $a(F)=0$ whenever $\operatorname{dim} F \neq r$.

What should especially be observed is that this condition is completely independent of the particular polytope $P$ above on which $a$ may be realized as a weight. The fact that $\mathcal{N}(P)$ is a refinement of $\mathcal{N}(Q)$ if $Q \preceq P$ also reinforces this perception; the discussion in Section 2 then shows that any given set of weights may be thought of as elements of $\Omega(P)$ for some simple polytope $P$. In spite of this observation, we shall continue to work with weights on actual polytopes. There is a straightforward reason: the various constructions we describe are firmly rooted in the geometry of polytopes, and the reader's intuition will, it is hoped, be reinforced from this viewpoint. However, all computational details will readily be seen to work at the abstract level, and so we try to refrain from tedious emphasis of this in what we do below.

We end with a brief remark. We may identify $a \in \Omega_{r}(P)$ with a (row) vector $a:=$ $\left(a(F) \mid F \in \mathcal{F}^{r}(P)\right)$, indexed by the $f_{r}(P)$ faces $F \in \mathcal{F}^{r}(P)$, where $f_{j}(P)$ denotes the number of $j$-faces of $P$ for $j=0, \ldots, d$. We denote by $U_{r, r+1}$ the $f_{r}(P) \times d f_{r+1}(P)$ matrix whose "entries" are the $1 \times d$ blocks $u_{F, G}$, with $F \in \mathcal{F}^{r}(P)$ and $G \in \mathcal{F}^{r+1}(P)$; here, conventionally, we write $u_{F, G}:=o$ if $F$ is not a facet of $G$. The condition for $a \in \Omega_{r}(P)$ is then just $a U_{r, r+1}=o$, so that $a \in \operatorname{ker} U_{r, r+1}$.

In [9], Lee has suggested the following pretty interpretation of $\operatorname{ker} U_{r, r+1}^{\top}$; the relationship between these two kernels is analogous to that between affine stresses and infinitesimal motions in the dual context of simplicial polytopes (see [8] and [17]). Changing indices, an element of $\operatorname{ker} U_{r-1, r}^{\top}$ associates a vector $v(F) \in \mathbb{E}^{d}$ with each $F \in \mathcal{F}^{r}(P)$, such that

$$
\sum_{F \in \mathcal{F}^{r}(F)}\left\langle u_{J, F}, v(F)\right\rangle=0
$$

for each $J \in \mathcal{F}^{r-1}(P)$. We may now think of $v(F)$ as a flow in $F$; observe that only the part of $v(F)$ which is parallel to $F$ plays an effective rôle. The term $\left\langle u_{J, F}, v(F)\right\rangle$ is that part of the flow which moves over the facet $J$ of $F$, if we think of $v$ as moving the whole $r$-volume of $F$ parallel to $v(F)$. The equation thus represents conservation of flow at each $(r-1)$-face $J$; in other words, $v$ is a circulation on $\mathcal{F}^{r}(P)$. Notice also the way in which the Minkowski relation on an $r$-face $F$ is involved in this interpretation; it ensures that the same amount of flow leaves $F$ as enters it. 


\section{Coherent Subdivisions}

Much of our discussion depends upon the different subdivisions of one polytope which arise from it as the union of certain projected faces of another, and the relationships between them. The appropriate context is that of fibre polytopes, as introduced in [3], although we do not need all the machinery of that paper.

Let $P$ be a $d$-polytope and let $Q$ be an $n$-polytope, such that $P$ is the image of $Q$ under some linear mapping $\varphi$. It is often convenient to take $Q \subseteq \mathbb{E}^{n}$ and $P \subseteq \mathbb{E}^{d}$, so that the linear mapping is $\varphi: \mathbb{E}^{n} \rightarrow \mathbb{E}^{n}$. Then $P$ will admit many subdivisions into $d$-polytopes which are images under $\varphi$ of faces of $Q$; certain of these are constructed as follows (compare the much earlier discussion in [18]).

The inverse image of a point $x \in P$ determines a section or fibre $Q(x):=Q \cap\{x\} \varphi^{-1}$ of $Q$, which is $(n-d)$-dimensional when $x \in \operatorname{int} P$, the interior of $P$. We call such a fibre general if $x \notin G \varphi$ when $G \in \mathcal{F}(Q)$ satisfies $\operatorname{dim} G<d$; in this case, each $m$-face of $Q(x)$ is of the form $G \cap\{x\} \varphi^{-1}$ for some $G \in \mathcal{F}^{d+m}(Q)$. These general fibres fall into finitely many strong isomorphism classes, determined by the regions into which $P$ is divided by the images $G \varphi$ of the $(d-1)$-faces $G$ of $Q$. Note that a special fibre $Q(x)$ (that is, one which is not general) is the limit of general fibres $Q\left(x^{\prime}\right)$ for which $Q(x) \preceq Q\left(x^{\prime}\right)$.

Let $\bar{Q}$ be the Minkowski sum of one fibre chosen from each such strong isomorphism class. After a suitable translation, we may regard $\bar{Q}$ as lying in $\operatorname{ker} \varphi$. Then $\bar{Q}$ is strongly isomorphic to the fibre polytope of the projection $\varphi$, whose definition is

$$
\operatorname{Fib}(Q, \varphi):=\frac{1}{\operatorname{vol}_{d}(P)} \int_{P} Q(x) d x
$$

the integral is well defined, since it can be described alternatively as the polytope whose support functional is the corresponding average of the support functionals of the fibres. We therefore stretch terminology a little, and refer to $\bar{Q}$ itself as the fibre polytope of the projection $\varphi$, and denote it by the same symbol $\operatorname{Fib}(Q, \varphi)$. We formulate things in this way, because then the concept extends to the similar situation where we work in spaces over an arbitrary ordered field instead of $\mathbb{R}$.

Now $\operatorname{Fib}(Q, \varphi)$ determines subdivisions of $P$ in the following way. Pick any (unit) normal vector $v \in \operatorname{ker} \varphi$. Then $v$ gives a face $Q(x)_{v}$ of each fibre $Q(x)$. Over each strong isomorphism class $\mathcal{K}$ of fibres of $Q$, this face $Q(x)_{v}$ is the intersection of $\{x\} \varphi^{-1}$ with the same face of $Q$, which we denote by $G(\mathcal{K}, v)$. The subdivision of $P$ induced by $v$ then consists of the polytopes, $G(\mathcal{K}, v) \varphi$. It is clear that this choice does indeed induce a subdivision of $P$, which we denote by $\mathcal{D}(v)$. A subdivision of $P$ which arises in this way is additionally called coherent (we follow here the terminology of [3], if not always the notation).

We begin with an obvious remark.

Lemma 4.1. If $\operatorname{Fib}(Q, \varphi)_{v}$ is a $k$-face of $\operatorname{Fib}(Q, \varphi)$, then the components of $\mathcal{D}(v)$ consist of the images of faces of $Q$ of dimension at most $d+k$. 
Proof. The reason is that the corresponding face $Q(x)_{v}$ of a general fibre $Q(x)$ also has dimension at most $k$, and this is the intersection of $\{x\} \varphi^{-1}$ with a face of $Q$ of dimension at most $d+k$.

In particular, we have:

Corollary 4.2. The coherent subdivisions of $P$ are in one-to-one correspondence with the non-empty faces of $\operatorname{Fib}(Q, \varphi)$. When one face of $\operatorname{Fib}(Q, \varphi)$ is contained in another, then the first subdivision is a refinement of the second.

The finest coherent subdivisions thus correspond to vertices of $\operatorname{Fib}(Q, \varphi)$, and are called tight; these are the ones which most interest us in what follows. The subdivision corresponding to $\operatorname{Fib}(Q, \varphi)$, regarded as a face of itself, is the trivial one.

A special case occurs frequently in our later discussion. When $n=d+1$, there are two tight subdivisions of $P, \operatorname{since} \operatorname{Fib}(Q, \varphi)$ is a line segment. In this case, $\operatorname{ker} \varphi=\operatorname{lin}\{v\}$ for some unit vector $v$. One tight subdivision $\mathcal{D}(v)$ then consists of the images $G \varphi$ of those facets $G$ of $Q$ for which $y+\mu v \notin Q$ for each $y \in G$ and $\mu>0$; these facets form what we call the upper surface of $Q$, relative to $v$ (or, more loosely, to $\varphi$ ). The other tight subdivision is $\mathcal{D}(-v)$, arising from the analogously determined lower surface of $Q$.

We also need to look at a subdivision $\mathcal{D}:=\mathcal{D}(v)$ which corresponds to an edge $E$ of $\operatorname{Fib}(Q, \varphi)$, giving the transition from one tight subdivision to another. By Lemma 4.1, the components of $\mathcal{D}$ are images $G \varphi$ of faces $G$ of $Q$ with $\operatorname{dim} G \leq d+1$. When $\operatorname{dim} G=d+1$, then $G \varphi$ is doubly covered by images of $d$-faces of $Q$, namely those arising from the upper and lower surfaces of $G$ relative to $\varphi$. The tight subdivisions arising from the vertices of $E$ then have $G \varphi$ replaced by the images of one or another of these two surfaces.

We end the section by describing how we may identify the faces $G$ of $Q$ given by the vector $v$ above, which contribute to a coherent subdivision $\mathcal{D}(v)$ of $P=Q \varphi$. It is now preferable to take $\varphi: \mathbb{E}^{n} \rightarrow \mathbb{E}^{d}$. Let $G \in \mathcal{F}(Q)$ be such that $G \varphi$ is a polytope in $\mathcal{D}(v)$; we do not suppose that $\operatorname{dim}(G \varphi)=d$. We need only concern ourselves with a fibre $Q(x)$ such that $x \in \operatorname{relint}(G \varphi)$, where relint denotes the relative interior. We see that $G$ is such that $Q(x)_{v} \supseteq G \cap Q(x)$. Then $v \in N(G \cap Q(x), Q(x))$ implies that $v+w \in N(G, Q)$ for some $w \in(\operatorname{ker} \varphi)^{\perp}$; to see this, observe that a hyperplane in $\{x\} \varphi^{-1}$ which supports $Q(x)$ is contained in one in $\mathbb{E}^{n}$ which supports $Q$, and this will necessarily contain $G$, since $\{x\} \varphi^{-1} \cap$ relint $G \neq \emptyset$. Since the argument is reversible, there follows:

Lemma 4.3. A face $G$ of $Q$ is such that $G \varphi \in \mathcal{D}(v)$ if and only if

$$
\left(v+(\operatorname{ker} \varphi)^{\perp}\right) \cap N(G, Q) \neq \emptyset .
$$

The criterion for a tight subdivision refines Lemma 4.3.

Theorem 4.4. Let $P \in \mathbb{E}^{d}$ and $Q \in \mathbb{E}^{n}$ be polytopes, and let $\varphi: \mathbb{E}^{n} \rightarrow \mathbb{E}^{d}$ be a linear mapping such that $P=Q \varphi$. The coherent subdivision $\mathcal{D}(v)$ arising from a vector $v \in \operatorname{ker} \varphi$ is tight if and only if

$$
\left.(v+\operatorname{ker} \varphi)^{\perp}\right) \cap \operatorname{relint} N(G, Q)=\emptyset
$$


whenever $G \in \mathcal{F}(Q)$ with $\operatorname{dim} G>d$. In this event,

$$
\mathcal{D}(v)=\left\{G \varphi \mid G \in \mathcal{F}(Q) \text { and }\left(v+(\operatorname{ker} \varphi)^{\perp}\right) \cap N(G, Q) \neq \emptyset\right\}
$$

\section{Linear Mappings}

This section is devoted to a core result, on which much of the subsequent discussion is based.

Theorem 5.1. A linear mapping between vector spaces induces a corresponding linear mapping between their weight spaces.

We establish the result for the weight spaces of polytopes. However, since the description of tight coherent subdivisions can be formulated in terms of normal cones, it will be clear that the definition of the linear mapping will not actually depend on the polytope on which any particular weight is carried.

We employ the notation of Section 4 , and assume that $P \in \mathbb{E}^{d}$ and $Q \in \mathbb{E}^{n}$ are polytopes with $P=Q \varphi$ for some linear mapping $\varphi: \mathbb{E}^{n} \rightarrow \mathbb{E}^{d}$. However, we begin with a very important observation.

The definition of weight appears to depend on the euclidean structure (that is, on the inner product and norm). In fact, an alternative picture makes it clear that this is not the case, and provides a useful insight into several results we prove below. The case $n=r+1$ and $d=r$ illustrates what is happening in sufficient generality. As we have seen in Section 4, there are two tight subdivisions of $P$, which arise from the upper and lower surfaces of $Q$ relative to a unit vector $v$ which spans the kernel of $\varphi$. If $u:=u_{G, Q}$, the outer normal to $Q$ at its facet $G$, then $G$ is upper or lower according to whether $\langle u, v\rangle>0$ or $\langle u, v\rangle<0$.

The Minkowski relation for an $r$-weight $a$ on $Q$ generalizes the case of $r$-volume vol $_{r}$. It just says that the total areas of the two coverings of $P$ induced by the subdivisions coincide with $\operatorname{vol}_{r}(P)$; note that the scaling factor of the area of the facet $G$ above under the projection on the hyperplane $v^{\perp}$ in $\mathbb{E}^{r+1}$ orthogonal to $v$ is $|\langle u, v\rangle|$. Thus the Minkowski relation for a general weight $a$ merely substitutes $a(F)$ for $\operatorname{vol}_{r}(G)$. However, it is clear that an oblique projection will preserve the relative scalings of these $r$-volumes; that is, it will multiply the existing scalings $|\langle u, v\rangle|$ by some fixed additional factor. Now the picture is a purely linear one, since linear mappings are equivalent to oblique projections.

We thus deduce a linear version of the Minkowski relation. Continuing with the notation above, for each $r$-face $G$ of $Q$, let $\gamma_{G, \varphi} \geq 0$ be defined by

$$
\gamma_{G, \varphi} \operatorname{vol}_{r}(G):=\operatorname{vol}_{r}(G \varphi)
$$

It is clear that $\gamma_{G, \varphi}$ really depends only on the linear subspace parallel to $G$; it is just $\operatorname{vol}_{r}(C \varphi)$ when $C$ is a unit $r$-cube in this subspace. Further, $\gamma_{G, \varphi}=0$ when $\varphi$ is singular on $G$. Then we have: 
Lemma 5.2. If $\varphi$ is a linear mapping on $Q$ with a one-dimensional kernel, and if $a \in \Omega_{r}(Q)$, then

$$
\sum_{G \text { upper for } \varphi} \gamma_{G, \varphi} a(G)=\sum_{G \text { lower for } \varphi} \gamma_{G, \varphi} a(G),
$$

where upper and lower are relative to some vector which spans $\operatorname{ker} \varphi$.

The same circle of ideas leads to another useful observation.

Lemma 5.3. Let $Q$ be an $(r+1)$-polytope, and let $a \in \Omega_{r}(Q)$. If $\varphi$ is a linear mapping which is one-to-one on $Q$, define $a \varphi: \mathcal{F}^{r}(Q \varphi) \rightarrow \mathbb{R}$ by

$$
(a \varphi)(G \varphi):=\gamma_{G, \varphi} a(G)
$$

Then $a \varphi \in \Omega_{r}(Q \varphi)$.

Proof. The proof results from applying $\varphi$ to the picture of the Minkowski relation generalized to oblique projections. The numbers $\gamma_{G, \varphi}$ give the relative scalings of all $r$-volumes involved in an oblique projection, and thus the Minkowski relation is preserved.

We are now in a position to discuss the general result. Let $\varphi$ be a linear mapping, let $Q$ be a polytope, and let $a \in \Omega_{r}(Q)$. We then define $a \varphi: \mathcal{F}^{r}(Q \varphi) \rightarrow \mathbb{R}$ by generalizing the idea of Lemma 5.3. Let $F \in \mathcal{F}^{r}(P)$, with $P:=Q \varphi$. Choose some tight coherent subdivision of $P$ induced by $\varphi$; this induces a tight coherent subdivision $\mathcal{D}$, say, of $F$ also. Define

$$
(a \varphi)(F):=\sum_{G \varphi \in \mathcal{D}} \gamma_{G, \varphi} a(G),
$$

with $\gamma_{G . \varphi}$ as above. We first have:

Lemma 5.4. The definition of $a \varphi$ is independent of the subdivision $\mathcal{D}$.

Proof. We can write the definition as

$$
(a \varphi)(F)=\sum_{G \varphi \in \mathcal{D}}(a \varphi)(G \varphi),
$$

again using the convention of Lemma 5.3. We change the subdivision $\mathcal{D}$ by moving along an edge-path of the fibre polytope $F i b(Q, \varphi)$. In moving from one vertex to an adjacent one, we saw in Section 4 that the subdivision of $F$ alters through interchanging, for one or more $(r+1)$-faces $J$ of $Q$, the image of the upper surface of $J$ under $\varphi$ with the image of the lower surface. Lemma 5.2 applied to such a face $J$ shows that the terms in the sum above arising from the upper surface equal those coming from the lower surface, which proves the result.

For the other part of Theorem 5.1, we have: 
Lemma 5.5. The image a $\varphi$ is a weight.

Proof. Consider a (tight) subdivision $\mathcal{D}$ of an $(r+1)$-face $F$ of $P$. Each $(r+1)$-polytope $G \varphi \in \mathcal{D}$ satisfies the Minkowski relation, by Lemma 5.3. However, on an interior $r$-face $J \varphi$ in $\mathcal{D}$, with $J \in \mathcal{F}^{r}(Q)$, the terms in the Minkowski relations involving $J \varphi$ for the two $(r+1)$-dimensional components $G \varphi$ which contain it are equal and opposite in sign, because the corresponding unit outer normals $u_{J \varphi, G \varphi}$ are. Hence, if we sum the Minkowski relation over all these polytopes $G \varphi$, we end up with the Minkowski relation for $a \varphi$ on $F$ itself, as wanted.

Although we have formulated everything in terms of weights on polytopes, Theorem 4.4 makes it clear that the definition of the image $a \varphi$ of a weight $a$ under a linear mapping $\varphi$ can be expressed abstractly, so that $a \varphi$ does not depend on $a$ belonging to $\Omega(Q)$ for any particular polytope $Q$.

We end the section with an obvious remark.

Theorem 5.6. If $\varphi$ and $\psi$ are linear mappings for which the composition $\varphi \psi$ is defined, then, for any appropriate weight $a$,

$$
a(\varphi \psi)=(a \varphi) \psi
$$

\section{The Weight Algebra}

The object of this section is to define an algebra structure on weights. More specifically, given $a \in \Omega_{r}(P)$ and $b \in \Omega_{s}(Q)$, with $P, Q$ both polytopes in $\mathbb{E}^{d}$, we define a commutative and associative product $a b$, and show that the result belongs to $\Omega_{r+s}(P+Q)$. It will follow from the definition and the description of the induced subdivisions of $P+Q$ that the product is really one of elements of $\Omega$. Later we are most interested in the case that $P=Q$ is a (simple) $d$-polytope, so that $P+P=2 P$; we have observed in Section 3 that $\Omega(P)=\Omega(2 P)$ in a natural way.

The multiplication will be induced by Minkowski addition; we proceed through the direct product.

Lemma 6.1. Weights $a \in \Omega_{r}(P)$ and $b \in \Omega_{s}(Q)$ induce a weight $a \times b \in \Omega_{r+s}(P \times Q)$.

Proof. First, recall the convention in Section 3 about an $r$-weight $a$, that we write $a(F)=0$ if $\operatorname{dim} F \neq r$. We then define $a \times b$ by

$$
(a \times b)(F \times G):=a(F) b(G),
$$

for $F \in \mathcal{F}(P)$ and $G \in \mathcal{F}(Q)$. We call $a \times b$ the direct product of $a$ and $b$. The Minkowski relation thus needs to be checked only on two kinds of faces-a product of an $r^{\prime}$-face of $P$ and an $s^{\prime}$-face of $Q$, with $r^{\prime}=r+1$ and $s^{\prime}=s$, or with $r^{\prime}=r$ and $s^{\prime}=s+1$. By symmetry, it is enough to consider the former case. If $F^{\prime}$ is an $(r+1)$-face 
of $P$ and $G$ an $s$-face of $Q$, then the unit normal vector to $F^{\prime} \times G$ at a facet $F \times G$ is just $\left(u_{F, F^{\prime}}, o\right)$, where $u_{F, F^{\prime}}$ is that to $F^{\prime}$ at $F$. The Minkowski relation for $F^{\prime}$, namely

$$
\sum_{F} a(F) u_{F, F^{\prime}}=o,
$$

then at once implies

$$
\sum_{F}(a \times b)(F \times G) u_{F \times G . F^{\prime} \times G}=\sum_{F} a(F) b(G)\left(u_{F, F^{\prime}}, o\right)=(o, o),
$$

so that $a \times b \in \Omega_{r+s}(P \times Q)$, as claimed.

We now come to our main result.

Theorem 6.2. If $a \in \Omega_{r}(P)$ and $b \in \Omega_{s}(Q)$, then there is an associative and commutative product $a b \in \Omega_{r+s}(P+Q)$.

Proof. The sum mapping $\sigma_{d}: \mathbb{E}^{d} \times \mathbb{E}^{d} \rightarrow \mathbb{E}^{d}$ is defined by

$$
(x, y) \sigma_{d}:=x+y,
$$

for $x, y \in \mathbb{E}^{d}$. We then define $a b$ by

$$
a b:=(a \times b) \sigma_{d} .
$$

This is clearly well defined by Theorem 5.1, and is obviously commutative since Minkowski addition is also. For the associativity, we write $\varphi \times \psi$ for the linear mapping obtained by applying $\varphi$ and $\psi$ separately to the terms of a product of linear spaces, and $\iota_{d}$ for the identity mapping on $\mathbb{E}^{d}$, and use

$$
\left(\sigma_{d} \times \iota_{d}\right) \sigma_{d}=\left(\iota_{d} \times \sigma_{d}\right) \sigma_{d}
$$

applied to $\mathbb{E}^{d} \times \mathbb{E}^{d} \times \mathbb{E}^{d}$. Using Theorem 5.6, the induced homomorphism applied to a product $(a \times b) \times c=a \times(b \times c)$ of weights leads to $(a b) c=a(b c)$, as required.

Before we go further, we see how we actually multiply two weights. We first consider a special case, when $r+s=d$. If $\operatorname{dim}(P+Q)<d$, the product will be zero anyway, so we suppose that $\operatorname{dim}(P+Q)=d$. We apply the technique of Section 4; a tight induced subdivision $\mathcal{D}$ expresses $P+Q$ as a union of direct sums $F+G$, with $F, G$ faces of complementary dimensions of $P, Q$, respectively. With $\operatorname{dim} F=r$ and $\operatorname{dim} G=s$, there is a constant $v_{F, G}$ which depends only on the translation classes of their affine hulls, such that

$$
v_{F, G} \operatorname{vol}_{r}(F) \operatorname{vol}_{s}(G):=\operatorname{vol}_{d}(F+G) ;
$$

$v_{F, G}$ is the volume obtained by replacing $F$ and $G$ by unit cubes of the appropriate dimensions in their affine hulls. 
To calculate $a b$, with $a \in \Omega_{r}(P)$ and $b \in \Omega_{s}(Q)$, we now substitute $a(F)$ for $\operatorname{vol}_{r}(F)$ and $b(G)$ for $\operatorname{vol}_{s}(G)$ for each such direct sum $F+G$ which occurs in the subdivision $\mathcal{D}$, and take the total over all of them. That is, we have

$$
(a b)(P)=\sum_{F+G \in \mathcal{D}} \nu_{F, G} a(F) b(G) .
$$

The polytopes $F+G$ which occur in $\mathcal{D}$ can be deduced from the criteria of Theorem 4.4 (see also [1] and [15]). In $\mathbb{E}^{d} \times \mathbb{E}^{d}$, the normal vector concerned is of the form $(v,-v)$ for some nonzero vector $v \in \mathbb{E}^{d}$. The subspace $(\operatorname{ker} \varphi)^{\perp}$ is now $\left\{(x, x) \mid x \in \mathbb{E}^{d}\right\}$, and the conditions on $(\operatorname{ker} \varphi)^{\perp}$ (or on $v$ ) reduce to relint $N(F, P) \cap \operatorname{relint}(N(G, Q)-v)=\emptyset$ whenever $F \in \mathcal{F}(P)$ and $G \in \mathcal{F}(Q)$ are such that $\operatorname{dim} F+\operatorname{dim} G>d$. If this holds, then the polytopes $F+G \in \mathcal{D}(v)$ are those for which $\operatorname{dim} F+\operatorname{dim} G=d$, and $N(F, P) \cap(N(G, Q)-v) \neq \emptyset$. Since these criteria depend on the normal cones to the faces of the polytopes rather than on the polytopes themselves, we conclude that the product is really defined on $\Omega_{r} \otimes \Omega_{s}$. For convenience, though, we usually work in suitable subspaces $\Omega_{r}(P)$.

More generally, if $r+s<d$, the value that $a b$ assigns to an $(r+s)$-face of $P+Q$ is that obtained by performing the above calculation in that face, using some tight induced subdivision. Of course, $a b=0$ if $r+s>d$.

The multiplication defined above is an algebra multiplication; it is clear that $(\lambda a) b=$ $\lambda(a b)=a(\lambda b)$ for any weights $a$ and $b$ and scalar $\lambda$. Moreover, the multiplication is also distributive over addition, because ordinary multiplication of numbers is. With these remarks, we may summarize the above discussion in:

\section{Theorem 6.3.}

(a) On any polytope $P$, the weight space $\Omega(P)$ is a graded algebra.

(b) The space $\Omega$ of all weights is a graded algebra.

It is easily seen that the identity in $\Omega$ is $1 \otimes\left\langle\mathbb{E}^{d}\right\rangle \in \Omega_{0}$. This corresponds to the 0 -weight $i_{Q} \in \Omega_{0}(Q)$ for any polytope $Q$, given by $i_{Q}(V)=1$ for each vertex $V$ of $Q$. Notice that multiplication by $i_{Q}$ will induce an injection $i_{Q}: \Omega(P) \hookrightarrow \Omega(P+Q)$ for any other polytope $P$. In Lemma 2.1 we saw that a polytope in $\mathbb{E}^{d}$ is a summand of some simple $d$-polytope. There then follows:

Theorem 6.4. Any given weight in $\Omega$ can be regarded as an element of $\Omega(P)$ for some simple $d$-polytope $P$.

\section{Homomorphisms}

We have already used the fact that linear mappings between spaces induce linear mappings on the corresponding weights. We now show that, in fact, there is an induced algebra homomorphism. Another such homomorphism is induced by restriction of weights to a face of a polytope.

We begin with linear mappings. 
Theorem 7.1. Let $\varphi: \mathbb{E}^{n} \rightarrow \mathbb{E}^{d}$ be a linear mapping, and let $a, b \in \Omega\left(\mathbb{E}^{n}\right)$. Then $(a b) \varphi=(a \varphi)(b \varphi)$.

Proof. As in Section 6, $\varphi$ induces a linear mapping $\varphi \times \varphi: \mathbb{E}^{n} \times \mathbb{E}^{n} \rightarrow \mathbb{E}^{d} \times \mathbb{E}^{d}$, defined by $(x, y) \varphi:=(x \varphi, y \varphi)$ for $x, y \in \mathbb{E}^{n}$. Further,

$$
\sigma_{n} \varphi=(\varphi \times \varphi) \sigma_{d}
$$

with the notation introduced in Section 6. It is clear that the product weights satisfy

$$
(a \times b)(\varphi \times \varphi)=(a \varphi) \times(b \varphi)
$$

the result now follows from the commutation with the sum mappings, using Theorem 5.6.

We have an immediate consequence.

Corollary 7.2. Linear mappings induce algebra homomorphisms on the corresponding weight spaces.

We now come to restrictions. Here, it is definitely better to think of weights as sitting on the faces of polytopes. If $P \in \mathcal{P}$ and $F \in \mathcal{F}(P)$, then a weight $a \in \Omega(P)$ clearly gives a restriction $\left.a\right|_{F}$ to the faces of $P$ contained in $F$.

Theorem 7.3. Restriction of weights to a face of a polytope is an algebra homomorphism.

Proof. This result is transparent, since restriction is clearly a vector space homomorphism, and calculation of products of weights is, in any case, carried out intrinsically.

It will not generally be the case that $\Omega(F)=\left.\Omega(P)\right|_{F}$ for $F \in \mathcal{F}(P)$. The simplest example is given by a square pyramid. For ease of explanation let $P$ be a pyramid which is a half-octahedron, with base the square $F$. The 1-weights on the three edges of a triangular face of $P$ must be equal, whence $\operatorname{dim} \Omega_{1}(P)=1$. However, $F$ is a polygon with four edges, so that $\operatorname{dim} \Omega_{1}(F)=2$ (compare Section 3 ).

\section{The First Weight Space}

For several reasons we need an alternative way to perform multiplication by an element of $\Omega_{1}$. In preparation for this, we discuss the first weight space $\Omega_{1}(P)$ for an arbitrary polytope $P$, and then consider further the special case when $P$ is simple.

First, suppose that $P^{\prime}$ is another polytope, such that $P^{\prime} \simeq P$. Then $P^{\prime}$ naturally corresponds to an element $p^{\prime} \in \Omega_{1}(P)$, with the weight $p^{\prime}(E)$ on an edge of $P$ just the length of the corresponding edge of $P^{\prime}$. Conversely: 
Lemma 8.1. A positive weight $p^{\prime} \in \Omega_{1}(P)$ corresponds to a polytope $P^{\prime} \simeq P$, which is unique up to translation.

Proof. In saying that $p^{\prime}$ is positive, we obviously mean that $p^{\prime}(E)>0$ for each $E$ of $P$. For the proof, we follow the argument of Section 15.1 of [5]. The edge $E^{\prime}$ of $P^{\prime}$ corresponding to an edge $E$ of $P$ will have length $p^{\prime}(E)$. To each vertex $w$ of $P$ we associate a vertex $w^{\prime}$ of $P^{\prime}$, in the following way. Fix one vertex $v^{\prime}$ of $P^{\prime}$, corresponding to an initial vertex $v$ of $P$. If the vertex $w$ of $P$ is adjacent to $v$, so that $E=\operatorname{conv}\{v, w\}$ is an edge of $P$, then the corresponding vertex $w^{\prime}$ of $P^{\prime}$ is defined by

$$
w^{\prime}:=v^{\prime}+\frac{p^{\prime}(E)}{p(E)}(w-v)
$$

where $p \in \Omega_{1}(P)$ similarly gives the length $p(E)$ of the edge $E$ of $P$. Hence, if $E^{\prime}:=\operatorname{conv}\left\{v^{\prime}, w^{\prime}\right\}$, then $E^{\prime}$ does have length $p^{\prime}(E)$.

We now iterate this process. If the vertex $w$ of $P$ is reached from $v$ by means of a chain of edges $E_{1}, \ldots, E_{m}$, then $w^{\prime}$ is reached from $v^{\prime}$ by means of the corresponding edges $E_{1}^{\prime}, \ldots, E_{m}^{\prime}$; each edge in the (directed) path can thus be thought of as a vector, defined as above. To see that $w^{\prime}$ is well defined, suppose that we have two different edge-paths from $v$ to $w$; we must show that they yield the same point $w^{\prime}$. Tracing one edge-path followed by the reverse of the other leads to a closed edge-path in $P$, and thus a vector arising from the directed path. We may contract this path to the vertex $v$ over the 2 -faces of $P$. The Minkowski relations for $p^{\prime}$ on these 2-faces ensure that each such contraction leaves the vector corresponding to the path unchanged; at $v$ it reduces to $o$, so that it was originally $o$, leading to the required conclusion.

To see that the convex hull $P^{\prime}$ of these vertices $w^{\prime}$ satisfies $P^{\prime} \simeq P$, we proceed by induction on the dimension. To each proper face $F$ of $P$ corresponds a strongly isomorphic polytope $F^{\prime}$; in particular, this is true when $F$ is a facet of $P$. Every vertex of $P$ which does not lie in $F$ is at the end of a path of edges, each directed away from the support hyperplane $H$ of $P$ which contains $F$. The corresponding edges of $P^{\prime}$ are directed away from the parallel hyperplane $H^{\prime}$ which contains $F^{\prime}$; the remaining vertices of $P^{\prime}$ thus lie on the same side of this hyperplane, so that $H^{\prime}$ supports $P^{\prime}$. It follows easily that $P^{\prime} \simeq P$, and $P^{\prime}$ is uniquely determined by the initial choice of $v^{\prime}$, as we had to show.

Under the correspondence $P^{\prime} \leftrightarrow p^{\prime}$, we clearly have

$$
\begin{aligned}
P^{\prime}+P^{\prime \prime} & \leftrightarrow p^{\prime}+p^{\prime \prime}, \\
\lambda P^{\prime} & \leftrightarrow \lambda p^{\prime} \quad \text { for } \quad \lambda \geq 0 .
\end{aligned}
$$

A general weight $y \in \Omega_{1}(P)$ is the difference of positive weights, since $y+\lambda p>0$ for a suitably large $\lambda$. Thus $y$ corresponds to a formal difference of polytopes in $\mathcal{K}(P)$; more specifically, since $\mathcal{K}(P)$ is a semigroup with cancellation under Minkowski addition, we can identify $y$ with an element of $\mathcal{P}_{T}(P)$, namely the group modulo translation which $\mathcal{K}(P)$ engenders. We recall the definition of this group (compare [11]): it has generators $(P, Q)$, with $P, Q \in \mathcal{K}(P)$, which satisfy the relations

$$
(P, Q):=\left(P^{\prime}, Q^{\prime}\right) \quad \Leftrightarrow \quad P+Q^{\prime}=P^{\prime}+Q+t,
$$


for some $t \in \mathbb{E}^{d}$, with addition defined by

$$
(P, Q)+\left(P^{\prime}, Q^{\prime}\right):=\left(P+P^{\prime}, Q+Q^{\prime}\right)
$$

Summarizing, we have:

Lemma 8.2. If $P$ is any polytope, then $\Omega_{1}(P) \cong \mathcal{P}_{T}(P)$.

For the remainder of this section, $P$ will be a simple $d$-polytope with $n$ facets. We can express $P$ in the form

$$
P=\left\{x \in \mathbb{E}^{d} \mid\left\langle x, u_{j}\right\rangle \leq \pi_{j}(j=1, \ldots, n)\right\},
$$

where $u_{j}:=u_{F_{j}, P}$ is the unit outer normal vector to $P$ at its facet $F_{j}$ for $j=1, \ldots, n$. In this context, we think of $U:=\left(u_{1}, \ldots, u_{n}\right)$ as a fixed set of normal vectors, and, as in Section $2, \pi_{j}:=\eta\left(P, u_{j}\right)$ is the (variable) support parameter of $P$ corresponding to $u_{j}$, for each $j$. The vector $p:=\left(\pi_{1}, \ldots, \pi_{n}\right) \in \mathbb{E}^{n}$ of these support parameters is called the support vector of $P$. In the previous discussion we did not distinguish between a polytope $P$ and one of its translates $P+t$ with $t \in \mathbb{E}^{d}$. This implies that we should identify the corresponding support vectors $p$ of $P$ and $\left(\pi_{1}+\left\langle t, u_{1}\right\rangle, \ldots, \pi_{n}+\left\langle t, u_{n}\right\rangle\right)$ of $P+t$, so that we must regard a support vector as lying in $\mathbb{E}^{n} / T$, where

$$
T:=\left\{\left(\left\langle t, u_{1}\right\rangle, \ldots,\left\langle t, u_{n}\right\rangle\right) \mid t \in \mathbb{E}^{n}\right\} .
$$

Note that an element of $T$ can be regarded as the support vector of a singleton point in the closure $\operatorname{cl} \mathcal{K}(P)$ of the strong isomorphism class $\mathcal{K}(P)$ of $P$. An arbitrary element of $\mathbb{E}^{n} / T$ is called a generalized support vector; for convenience, we continue to write a generalized support vector as an element of $\mathbb{E}^{n}$.

From the discussion in Section 2, the support vectors of polytopes in $\mathcal{K}(P)$ obviously behave as expected under Minkowski addition and non-negative scalar multiplication. Only certain generalized support vectors (even among those which are positive) correspond to polytopes in $\mathcal{K}(P)$. However, since $\mathcal{K}(P)$ consists of simple polytopes, any small enough perturbation of an actual support vector of a polytope in $\mathcal{K}(P)$ yields the support vector of another. There follows at once:

Lemma 8.3. If $P$ is a simple d-polytope, then $\mathcal{P}_{T}(P) \cong \mathbb{E}^{n} / T$.

Combining Lemmas 8.2 and 8.3 , we deduce:

Theorem 8.4. If $P$ is a simple d-polytope with $n$ facets, then the weight space $\Omega_{1}(P)$ is naturally isomorphic to $\mathbb{E}^{n} / T$, with $T$ the subspace of support vectors of points in $\operatorname{cl} \mathcal{K}(P)$.

We end the section with a remark about restrictions.

Lemma 8.5. If $P$ is a simple polytope and $F$ is a face of $P$, then $\Omega_{1}(F)=\left.\Omega_{1}(P)\right|_{F}$. 
Proof. Since $P$ is simple, any sufficiently small perturbation of the support parameters of $F$ will be induced by a suitable perturbation of those of $P$. The result follows at once.

\section{An Alternative Formula}

We are now in a position to discuss an alternative formulation of multiplication of a weight in $\Omega(P)$ by an element of $\Omega_{1}(P)$ when $P$ is simple. (In view of the remarks at the end of Section 3, this is not a serious restriction.) We follow the conventions of Section 8 throughout. We begin with a special case.

Theorem 9.1. If $y \in \Omega_{1}(P)$ and $a \in \Omega_{d-1}(P)$, then

$$
y a=\sum_{j=1}^{n} \eta_{j} a\left(F_{j}\right),
$$

where $y=\left(\eta_{1}, \ldots, \eta_{n}\right)$, regarded as a generalized support vector.

This expression generalizes one familiar in the theory of mixed volumes of polytopes (for instance, see Lemma 5.1.4 of [15]), wherefore we call it the asymmetric mixed volume calculation.

Proof. First note that the sum is well defined, since two choices for the generalized support vector differ by a vector of the form $\left(\left\langle t, u_{1}\right\rangle, \ldots,\left\langle t, u_{n}\right\rangle\right)$, and since

$$
\sum_{j=1}^{n}\left\langle t, u_{j}\right\rangle a\left(F_{j}\right)=0,
$$

from the Minkowski relations for $\Omega_{d-1}(P)$. We must therefore show that this expression is the same as that obtained when we use the original definition of the product.

It is enough to prove the theorem for a support vector of the form $e_{k}:=\left(\delta_{k 1}, \ldots, \delta_{k n}\right)$, with $\delta_{k j}$ the usual Kronecker delta function; we then extend by linearity. Regarded as a weight, if $E$ is an edge of $P$ which does not meet the facet $F_{k}$, then $e_{k}(E)=0$. The value that $e_{k}$ takes on an edge $E \subseteq F_{k}$ will not concern us, as we shall soon see. The remaining edges $E$ are those which meet $F_{k}$ in a single vertex; the value $e_{k}(E)$ is the length of the intercept of a line in the direction of $E$ with a slab between two hyperplanes with normal $u_{k}$ at unit distance apart. (The reader may find the following picture helpful. Replace $P$ by a sufficiently large scalar multiple, so that $e_{k}=p^{\prime}-p$ for some $P^{\prime} \in \mathcal{K}(P)$ also; we adopt the conventions used above. Then $e_{k}(E)=p^{\prime}(E)-p(E)$; of course, $p(E)$ and $p^{\prime}(E)$ are just the edge-lengths of $E$ and the corresponding edge $E^{\prime}$ of $P^{\prime}$.)

We now calculate the product $y a$, by means of a suitably chosen induced subdivision $\mathcal{D}(v)$ of $P+P$. We take the general vector $v$ to be a small perturbation of $u_{k}$. Then $v$ will have the following properties. Let

$$
C:=\bigcup_{w \in \operatorname{vert} F_{k}} N(w, P),
$$


the union of the normal cones to the vertices $w$ of $P$ in $F_{k}$; then $C \subset$ int $C-v$. Further, the half-line

$$
R:=N\left(F_{k}, P\right)-v=\operatorname{pos}\left\{u_{k}\right\}-v
$$

will intersect precisely one normal cone $N(E, P)$ to an edge $E$ of $P$ which meets $F_{k}$ in a single vertex; it will meet no normal cone to an edge of $F_{k}$ itself (because $R$ is parallel to such a cone), and we do not care about any other edges.

The usual calculation employing $\mathcal{D}(v)$ is now straightforward. In $\mathcal{D}(v)$, only one term contributes to the product, namely $E+F_{k}$ arising from $N(E, P) \cap\left(N\left(F_{k}, P\right)-v\right)$, for this particular edge $E$ above. This contribution is $1 \cdot a\left(F_{k}\right)=a\left(F_{k}\right)$, as required.

The general asymmetric mixed volume calculation works in a similar way. Recall that weight calculations on a face of a polytope are performed intrinsically. Thus, if $y \in \Omega_{1}(P)$ and $a \in \Omega_{r}(P)$ with $0 \leq r \leq d-2$, then we evaluate a term $(y a)(F)$ in the product $y a$, when $F$ is a $(r+1)$-face of $P$, within the face $F$. For our alternative procedure, we must then regard $y$ as a support vector in $F$; that is, for these purposes, $y$ then becomes the generalized support vector induced in $F$ (compare Lemma 8.5).

\section{Separation Properties}

In this section we conclude our discussion of the basic properties of the weight algebra $\Omega$. We begin with an important separation property of the spaces $\Omega_{r}$.

Theorem 10.1. If $r=0, \ldots, d$, then $\Omega_{d-r}$ separates $\Omega_{r}$.

Proof. What the theorem means is that if $a \in \Omega_{r}$ satisfies $a \neq 0$, then $b \in \Omega_{d-r}$ exists such that $a b \neq 0$. We prove it using Theorem 9.1. We may assume from Theorem 6.4 that $a \in \Omega_{r}(P)$, for some simple $d$-polytope $P$. We first consider the special case $r=d-1$. If $a \in \Omega_{d-1}(P)$ is non-zero, then, with the notation of Section 9 , we have $a\left(F_{k}\right) \neq 0$ for some $k=1, \ldots, n$. With $e_{k} \in \Omega_{1}(P)$ as in the proof of Theorem 9.1, we have $e_{k} a=a\left(F_{k}\right) \neq 0$, as we wanted.

For smaller $r$, we use induction on $d$. As before, we can regard $a \in \Omega_{r}$ as $a \in \Omega_{r}(P)$ for some simple $d$-polytope $P$. If $a \neq 0$, then we may choose some $F \in \mathcal{F}^{r+1}(P)$ such that $\left.a\right|_{F} \neq 0$. Since $P$ is simple, by Lemma 8.5 we have $\Omega_{1}(F)=\left.\Omega_{1}(P)\right|_{F}$. Hence, by the first part, an element $e \in \Omega_{1}(P) \subseteq \Omega_{1}$ exists such that $\left.(e a)\right|_{F}=\left.\left.e\right|_{F} a\right|_{F} \neq 0$. Thus $e a \neq 0$, which is the inductive step, and the theorem follows at once.

On inspection of the proof of Theorem 10.1, we see that we have actually proved a little more, namely that when $P$ is a simple $d$-polytope, then $\Omega_{r}(P)$ is separated by $\Omega_{1}(P)^{d-r}$, where the latter (following the usual convention) is the subspace of $\Omega_{d-r}$ generated by the $(d-r)$-fold products of elements of $\Omega_{1}(P)$. Now the dimension of a space cannot exceed that of one which separates it. Hence, interchanging $r$ and $d-r$ and using Theorem 10.1 twice (the latter time in this stronger form), a dimension count yields

$$
\operatorname{dim} \Omega_{r}(P) \leq \operatorname{dim} \Omega_{d-r}(P) \leq \operatorname{dim} \Omega_{1}(P)^{r} \leq \operatorname{dim} \Omega_{r}(P)
$$


All the dimensions must therefore be equal; we thus deduce that $\Omega_{r}(P)=\Omega_{1}(P)^{r}$, and hence:

Theorem 10.2. If $P$ is a simple polytope, then $\Omega(P)$ is generated as an algebra by $\Omega_{1}(P)$. Hence $\Omega$ is similarly generated by $\Omega_{1}$.

Observe that Theorem 10.2 does not hold in general for a non-simple polytope $P$. Indeed, if $d \geq 3$, and $P$ is a simplicial $d$-polytope with $n \geq d+2$ facets, then $\operatorname{dim} \Omega_{1}(P)=1$, but $\operatorname{dim} \Omega_{d-1}(P)=n-d \geq 2$.

Another useful consequence of the argument is:

Theorem 10.3. Let $P$ be a simple d-polytope. Then $\operatorname{dim} \Omega_{r}(P)=\operatorname{dim} \Omega_{d-r}(P)$ for each $r=0, \ldots, d$.

We can now also generalize Lemma 8.5. That lemma, and Theorem 10.2, imply:

Theorem 10.4. If $P$ is a simple polytope and $F \in \mathcal{F}(P)$, then $\Omega(F)=\left.\Omega(P)\right|_{F}$.

\section{Volumes of Faces}

We now consider the relationship between the $r$-volumes of the $r$-faces of a fixed polytope, when they are regarded as elements of $\Omega$. In particular, we establish that, in a sense, we have actually been talking about volumes and mixed volumes all along.

If $P$ is a polytope in $\mathbb{E}^{d}$, and if $r=0, \ldots, d$, then $P$ induces an element $p_{r} \in$ $\Omega_{r}(P) \subseteq \Omega_{r}$ by $p_{r}(F):=\operatorname{vol}_{r}(F)$ for each $r$-face $F$ of $P$; we call this element the $r$-class of $P$. We write $[P]:=\sum_{r=0}^{d} p_{r}$, which we call the (total) class of $P$. (The terminology is intended to be reminiscent of that of [11].)

Theorem 11.1. Let $P$ be a polytope, and for each $r=0, \ldots, d$ let $p_{r}$ be its $r$-class. Then $p_{r}=(1 / r !) p_{1}^{r}$.

Proof. Let $P$ and $Q$ be any polytopes in $\mathbb{E}^{d}$. From the definition of the product and the way that it is related to volume, for any $k$ and any $\lambda, \mu \geq 0$, the $k$-class of $\lambda P+\mu Q$ is

$$
\sum_{r+s=k} \lambda^{r} \mu^{s} p_{r} q_{s}
$$

where $q_{s} \in \Omega_{s}$ is the $s$-class of $Q$. Note that $r$-volume is positive homogeneous of degree $r$. When $P=Q$, the $k$-class of $\lambda P+\mu P=(\lambda+\mu) P$ is $(\lambda+\mu)^{k} p_{k}$, and so taking $k=r+s$, comparing coefficients of $\lambda^{r} \mu^{s}$, and clearing fractions shows that

$$
r ! p_{r} \cdot s ! p_{s}=(r+s) ! p_{r+s}
$$

for all $r, s \geq 0$. An easy induction argument (with $s=1$ ) leads to the result.

As an aside, note the case $r=1$ and $s=d-1$ of the above relation. If, as in Section 8, we identify the 1-class $p:=p_{1} \in \Omega_{1}(P)$ of the not necessarily simple $d$-polytope $P$ 
with its support vector $\left(\pi_{1}, \ldots, \pi_{n}\right)$, and write $\alpha_{j}:=\operatorname{vol}_{d-1}\left(F_{j}\right)$ for $j=1, \ldots, n$, then from $p_{d}=(1 / d) p_{1} p_{d-1}$ we recover the familiar formula

$$
\operatorname{vol}_{d}(P)=\frac{1}{d} \sum_{j=1}^{n} \pi_{j} \alpha_{j}
$$

Using the notation $p:=p_{1}$, we see that we can express Theorem 11.1 in two suggestive ways.

Corollary 11.2. Let $P$ be a (non-empty) polytope in $\mathbb{E}^{d}$. If $p \in \Omega_{1}$ is the 1-class of $P$, then:

(a) $[P]=\exp p$;

(b) $p=\log [P]$.

It is often convenient (compare p. 94 of [11]) to abbreviate $\log [P]$ to $\log P$.

Theorems 10.2 and 11.1 together now have an important implication. The definition of the product of two weights generalizes the calculation of mixed volumes. Further, a mixed volume is a rational linear combination of volumes, and hence (replacing polytopes by suitable dilatates) an integer linear combination of such volumes. More specifically, $\Omega_{r}=$ $\Omega_{1}^{r}$ is generated by elements $p_{1}, \ldots, p_{r}$, with $p_{1}, \ldots, p_{r} \in \Omega_{1}$. Using the polarization formula

$$
p_{1} \cdots p_{r}=\frac{1}{r !} \sum_{s=1}^{r} \sum_{1 \leq j(1)<\cdots<j(s) \leq r}(-1)^{r-s}\left(p_{j(1)}+\cdots+p_{j(s)}\right)^{r}
$$

we deduce:

Lemma 11.3. For each $r=0, \ldots, d$, the $r$ th weight space $\Omega_{r}$ is generated by the elements $p^{r}$, with $p \in \Omega_{1}$. Similarly, if $P$ is a simple polytope, then $\Omega_{r}(P)$ is generated by the elements $p^{r}$, with $p \in \Omega_{1}(P)$.

Theorem 10.2, or its refined form Lemma 11.3, can now be interpreted as saying:

Theorem 11.4. A weight $a \in \Omega$ is a linear combination of volumes of faces of polytopes.

We end the section with a remark. In the proof of Theorem 5.1 we used tight coherent subdivisions of a polytope $P=Q \varphi$ induced by a linear mapping $\varphi$. However, it is now apparent that we do not need the subdivisions to be coherent, as long as they arise from projections of faces of $Q$ under $\varphi$. The reason is that any subdivision will give the correct answer for the projected weight if the original was ordinary volume (the projection will also be volume); the general case follows from Theorem 11.4. 


\section{The Polytope Algebra}

We end the discussion of the weight algebra $\Omega$ by considering the connexion between it and the polytope algebra $\Pi$ of [11]. Recall the basic definitions. The polytope algebra $\Pi$ as an abelian group has a generator $[P]$ for each $P \in \mathcal{P}$, with $[\emptyset]:=0$. These generators satisfy the relations (V) $[P \cup Q]+[P \cap Q]=[P]+[Q]$ whenever $P, Q \in \mathcal{P}$ are such that $P \cup Q \in \mathcal{P}$ also (the valuation property), and (T) $[P+t]=[P]$ whènever $P \in \mathcal{P}$ and $t \in \mathbb{E}^{d}$ (translation invariance). Further, $\Pi$ admits a (commutative) multiplication (M) $[P] \cdot[Q]:=[P+Q]$ for $P, Q \in \mathcal{P}$ (induced by Minkowski addition), and extended by linearity. Finally, the dilatation operator $\Delta$ acts on $\Pi$ by (D) $\Delta(\lambda)[P]:=[\lambda P]$ for $P \in \mathcal{P}$ and $\lambda \in \mathbb{R}$.

The main structure theorem for $\Pi$ is Theorem 1 of $[11]$.

Theorem 12.1. $\Pi$ is almost a graded algebra over $\mathbb{R}$, in the following sense:

(a) There is a direct sum decomposition $\Pi=\bigoplus_{r=0}^{d} \Pi_{r}$, with $\Pi_{0} \cong \mathbb{Z}$, the integers, and $\Pi_{r}$ a real vector space for $r=1, \ldots, d$ (with $\left.\Pi_{d} \cong \mathbb{R}\right)$.

(b) $\Pi_{r} \cdot \Pi_{s}=\Pi_{r+s}$ for $r, s \geq 0$ (with $\Pi_{k}:=\{0\}$ for $k>d$ ).

(c) $(\lambda a) b=\lambda(a b)=a(\lambda b)$ for $a, b \in Z_{1}:=\bigoplus_{r=1}^{d} \Pi_{r}$ and $\lambda \in \mathbb{R}$.

(d) $\Delta(\lambda) a=\lambda^{r}$ a for $a \in \Pi_{r}$ and $\lambda \geq 0$.

An easier proof than that in [11], establishing a more general result, is to be found in [14]. (Note that, in [11], we used the notation $\Xi_{r}$ instead of $\Pi_{r}$.) It is natural at this point to make $\Pi$ into a full algebra; we replace $\Pi_{0}$ by $\mathbb{R} \otimes \Pi_{0} \cong \mathbb{R}$.

The connexion between the polytope and weight algebras is provided by Theorem 2 of [11] and by [13].

Theorem 12.2. The mapping $P \mapsto \sum_{F} \operatorname{vol}(F) \otimes\langle N(F, P)\rangle$ induces an algebra isomorphism between $\Pi$ and $\Omega$.

The sum here extends over all faces $F$ of $P$, and the volume and class of normal cone are taken intrinsically (we employ the conventions of Section 3 ). Naturally, this identifies the classes $[P]$ of $P \in \mathcal{P}$ as defined here and in Section 11, and explains why we have employed the same notation for them.

Actually, all that was shown in [11] and [13] was that we have a vector space isomorphism, and as we remarked in Section 1, strictly speaking the argument of [13] is incomplete (we have repaired the omissions here). However, the fact that multiplication is preserved as well is a direct consequence of the weaker isomorphism, because in $\Pi$ multiplication also reduces to the mixed volume calculations.

In the cited papers, everything was done over an arbitrary ordered field. We pointed out in Section 1 that the same can be done for the weight algebra; the above isomorphism then carries over. 


\section{On the $g$-Theorem}

As we said in Section 1, the weight algebra $\Omega$ provides an easier approach to the necessity part of the $g$-theorem than that employed in [12] using the polytope algebra $\Pi$. Of course, in view of Section 12, we know that $\Omega \cong \Pi$, or, more specifically for these purposes, that $\Omega(P) \cong \Pi(P)$ for each simple polytope $P$. In any event, again as we said in Section 1 , in [12] we largely used weights in the proof.

We do not repeat large parts of [12] here, with just the appropriate changes of language. Thus this section must be read in conjunction with [12]; except in one instance, we merely provide signposts to the amendments.

First, there are some general remarks. Each occurrence in [12] of $\Pi$ (also in various qualified forms such as $\Pi(P)$ ) should be replaced by $\Omega$; similarly, the weight space $\Xi_{r}$ should become $\Omega_{r}$ (we have written $\Pi_{r}$ instead of $\Xi_{r}$ in Section 12). There is also a technical difference between our usage of $\mathcal{K}(P)$ in the two papers; in effect, in [12], we have factored out translations already, whereas here we have not.

Next, we may skip over much of the first few sections of [12]. We can discard Section 2 of [12] completely. All of Section 3 of [12] is also redundant, since in Section 8 we have avoided the use of the theory of representations of polytopes in establishing what results are needed. While flips (see below) were first formulated in terms of representations, more directly geometrical descriptions were given later in the section. Nevertheless, the reader may find comparison of the alternative approaches helpful; bear in mind that $p=\log P$ should then be thought of as the 1 -weight of a polytope $P$, determined by its edge-lengths (its identification as a support vector remains). All we need from Section 4 of [12] is that $h_{r}(P)$ is the number of vertices of type $r$ of a simple $d$-polytope $P$; the dimension $\operatorname{dim} \Omega_{r}(P)=h_{r}(P)$ of the weight space is found in Section 6 of [12], and the fact that $h_{r}(P)=h_{d-r}(P)$ follows from Theorem 10.3 of this paper. Similarly, Section 5 of [12] is also covered here, and so may be ignored (the lacuna mentioned in Section 1 as the starting point of this paper could have occurred in the proof of Lemma 5.3).

Before we proceed further, it is appropriate to reproduce the two main results, Theorems 7.3 and 8.2, from [12], in the language of this paper. The first gives the Lefschetz decomposition, which leads directly to the necessity part of the $g$-theorem. The second contains the Hodge-Riemann-Minkowski inequalities, which yield an inductive proof of the first theorem.

Theorem 13.1. Let $P$ be a simple d-polytope, and let $p:=\log P$. Then $p^{d-2 r} \Omega_{r}(P)=$ $\Omega_{d-r}(P)$ for $0 \leq r \leq \frac{1}{2} d$.

With this notation, the $r$ th primitive space $\tilde{\Omega}_{r}(P)$ of $\Omega(P)$ is defined by

$$
\tilde{\Omega}_{r}(P):=\left\{x \in \Omega_{r}(P) \mid p^{d-2 r+1} x=0\right\} .
$$

Theorem 13.2. If $P$ is a simple d-polytope, then for $0 \leq r \leq \frac{1}{2} d$ the quadratic form $(-1)^{r} p^{d-2 r} x^{2}$ is positive definite on $\tilde{\Omega}_{r}(P)$.

As we have clearly stated, we have no intention of reproducing the details of the proofs here. However, there is one place, namely in the proof of the core result [12, 
Lemma 11.7], where we went a little too quickly. We must set the background. We have two simple $d$-polytopes $P$ and $Q$, which are related by means of a flip, which corresponds combinatorially in the dual context to a bistellar operation. We could assume that the (unit) outer normal vectors $u_{1}, \ldots, u_{n}$ to the facets of the two polytopes coincide (with a minor exception which will emerge in the description), and are in linearly general position. We perform an $m$-flip to get from $P$ to $Q$, with $1 \leq m \leq \frac{1}{2}(d+1)$, if the following properties hold:

- There is a one-to-one correspondence $F_{j} \leftrightarrow G_{j}(j=1, \ldots, n)$ between the facets $F_{j}$ of $P$ and $G_{j}$ of $Q$ with normal vector $u_{j}$, except that, if $m=1$, then $F_{1}$ is missing.

- $F:=F_{m+1} \cap \cdots \cap F_{d+1}$ is an (m-1)-face of $P$ bounded (in its affine hull) by $F_{1}, \ldots, F_{m}$.

- $G:=G_{1} \cap \cdots \cap G_{m}$ is a $(d-m)$-face of $Q$, bounded by $G_{m+1}, \ldots, G_{d+1}$.

- If $J \subseteq\{1, \ldots, n\}$ with $J \nsubseteq\{1, \ldots, d+1\}$, then $\bigcap\left\{F_{j} \mid j \in J\right\}$ is a face of $P$ if and only if $\bigcap\left\{G_{j} \mid j \in J\right\}$ is a face of $Q$.

The faces $F$ of $P$ and $G$ of $Q$ are called their special faces.

Actually, as was shown in [12], we can take $u_{1}, \ldots, u_{d+1}$ to be the normal vectors (not all outer) to the facets of a regular $d$-simplex $\bar{S}$. In fact, $\bar{S}$ is bounded by the facet hyperplanes $H_{j}:=$ aff $G_{j}$ of $Q$ for $j=1, \ldots, d+1$; the outer facet normals to $\bar{S}$ are $-u_{1}, \ldots,-u_{m}, u_{m+1}, \ldots, u_{d+1}$. (We thus think of $\bar{S}$ as sharing the $(d-m)$-face $G$ with $Q$.) Each face of $\bar{S}$ is parallel to a unique face of $P$ which meets $F$ or of $Q$ which meets $G$ (possibly both), and conversely; faces of $\bar{S}$ also correspond to parallel faces of $P+Q$, but not uniquely to faces of $F+G$. This face of $P$ or $Q$ (in fact, here we only need the latter) is of kind $k$ if the corresponding face of $\bar{S}$ has $k$ vertices outside $G$. The intersection of facets $G_{j}$ of $Q$, with the $j$ running over a subset of $\{1, \ldots, d+1\}$, is of kind $k$ when exactly $k$ of $\{1, \ldots, m\}$ are missing from the subset.

The weight algebras $\Omega(P)$ and $\Omega(Q)$ are, of course, subalgebras of $\Omega(P+Q)$. When $m=1$, we have $P \preceq Q$; we can think of $Q$ as obtained from $P$ by slicing off a new facet $G_{1}$. In this case, $P+Q \simeq Q$, and $\Omega(P+Q)=\Omega(Q)$. When $m>1$, it is not hard to see that $P+Q$ is also a simple polytope; it has one new facet $F+G$ (the direct sum of the ( $m-1)$-simplex $F$ and $(d-m)$-simplex $G)$, whose unit outer normal vector we designate by $u_{0}$. (When $m=1$, we can make the identification $u_{0}:=u_{1}$.)

The difference between the weight spaces $\Omega_{r}(P)$ and $\Omega_{r}(Q)$ is carried on the $r$-faces of $\bar{S}$, or, more exactly, on the corresponding parallel $r$-faces of $P+Q$ which meet $F+G$. We are only interested here in the case $r \geq m$. Then the essentially unique positive $r$ weight $\bar{s}_{r} \in \Omega_{r}(\bar{S})$ transfers to $\Omega_{r}(Q)$. Its value on an $r$-face of $Q$ of kind $k$ which meets $G$ is numerically the same as that on the parallel $r$-face of $\bar{S}$, with a sign change $(-1)^{k}$.

We then have:

Lemma 13.3. There is an element $s:=s_{r} \in \Omega_{r}(P+Q)$, such that, if $1 \leq r \leq d-m$, then, for each $y \in \Omega_{r}(Q)$, there is a unique $x \in \Omega_{r}(P)$ and $\lambda \in \mathbb{R}$, such that $y=x+\lambda$ s.

By the way, this corrects a mistake in the statement of Theorem 11.2 of [12], where the restriction on $r$ is missing; the omission is obvious, on comparing this theorem with 
Theorem 11.3. Here, we wish to concentrate on the case $r \geq m$, when $\Omega_{r}(P)$ is a subspace of $\Omega_{r}(Q)$ of codimension 1 .

The problem in the proof of Lemma 11.7 is that crucial details were skated over. Slightly modified, Lemma 11.7 states:

Lemma 13.4. If $q:=\log Q$ and $s:=s_{r}$ with $m \leq r \leq \frac{1}{2} d$, then $(-1)^{m} q^{d-2 r} s^{2}>0$.

Proof. In [12] the range of $r$ was extended to $r \geq \frac{1}{2} m$; this is more general than we need (or is proved). The induction argument, reducing the proof to the case $d=2 r$, is perfectly correct. (This is the paragraph in Section 11 of [12] immediately preceding the statement of the lemma.) We prove Lemma 13.4 when $d=2 r$ by specifying a subdivision of $Q+Q$, which shows that only one pair $\left(G^{\prime}, G^{\prime \prime}\right)$ of faces of $Q$ can contribute to the product $s^{2}$ via a sum $G^{\prime}+G^{\prime \prime}$. Of course, only faces of $Q$ which meet the special face $G$ will be involved here.

By the assumptions made above, the normal vectors $u_{j}$ to the facets $G_{j}$ of $Q$ (with $j=1, \ldots, d+1$ ) are such that (up to a positive scaling of $u_{0}$ )

$$
u_{0}:=\sum_{j=1}^{m} u_{j}=\sum_{j=m+1}^{d+1} u_{j}
$$

(Actually, even if we do not apply a linear mapping to make $\bar{S}$ a regular simplex, we may always scale the normal vectors $u_{j}$ so that this relationship holds.) We choose the vector $v$ which gives the subdivision $\mathcal{D}(v)$ of $Q+Q$ to satisfy

$$
v=\sum_{j=0}^{d+1} \lambda_{j} u_{j}
$$

with $\lambda_{0}>0,0=\lambda_{1}<\cdots<\lambda_{m}$, and $\lambda_{m+1}>\cdots>\lambda_{d+1}=0$. We think of taking $v$ near $u_{0}$, so that we may choose $\lambda_{0}=1$ and all other $\lambda_{j}$ small. With a sufficiently random choice of the $\lambda_{j}$, this will also ensure that $v$ leads to a tight subdivision $\mathcal{D}(v)$. The $r$-weight $s$ is supported by those $r$-faces of $Q$ whose normal cone is spanned by $r$ of the vectors $u_{1}, \ldots, u_{d+1}$.

If the vector $u_{k}$ is the one excluded from an expression

$$
\text { relint pos } U^{\prime} \cap\left(\text { relint pos } U^{\prime \prime}-v\right) \neq \emptyset,
$$

with $U^{\prime} \cap U^{\prime \prime}=\emptyset$ and $U^{\prime} \cup U^{\prime \prime}=\left\{u_{1}, \ldots, u_{k-1}, u_{k+1}, \ldots, u_{d+1}\right\}$, then we may eliminate $u_{k}$ from the expression for $v$. If it were possible to have $k \in\{1, \ldots, m\}$, then

$$
\lambda_{0} u_{0}=-\lambda_{k}\left(\sum_{j=1}^{m} u_{j}\right)+\left(\lambda_{0}+\lambda_{k}\right)\left(\sum_{j=m+1}^{d+1} u_{j}\right)
$$

would follow. We would then have

$$
v=\sum_{j=1}^{m}\left(\lambda_{j}-\lambda_{k}\right) u_{j}+\sum_{j=m+1}^{d+1}\left(\lambda_{0}+\lambda_{k}+\lambda_{j}\right) u_{j},
$$


or, rearranging the sums,

$$
\sum_{j=1}^{k-1}\left(\lambda_{k}-\lambda_{j}\right) u_{j}=\sum_{j=k+1}^{m}\left(\lambda_{j}-\lambda_{k}\right) u_{j}+\sum_{j=m+1}^{d+1}\left(\lambda_{0}+\lambda_{k}+\lambda_{j}\right) u_{j}-v .
$$

The coefficients of the $u_{j}$ on each side are positive, and the expression for $v$ is unique (every $d$ vectors from $\left\{u_{1}, \ldots, u_{d+1}\right\}$ form a basis of $\mathbb{E}^{d}$ ), so that we conclude that we had to have $U^{\prime}=\left\{u_{1}, \ldots, u_{k-1}\right\}$ and $U^{\prime \prime}=\left\{u_{k+1}, \ldots, u_{d+1}\right\}$. Since $k \leq m$, this clearly would not allow $U^{\prime}$ to have $r \geq m$ elements.

We must therefore have $k \in\{m+1, \ldots, d+1\}$, when the rôles of the two sums are interchanged. That is, we now have (after similar calculations)

$$
v=\sum_{j=1}^{m}\left(\lambda_{0}+\lambda_{j}+\lambda_{k}\right) u_{j}+\sum_{j=m+1}^{d+1}\left(\lambda_{j}-\lambda_{k}\right) u_{j}
$$

yielding

$$
\sum_{j=k+1}^{d+1}\left(\lambda_{k}-\lambda_{j}\right) u_{j}=\sum_{j=1}^{m}\left(\lambda_{0}+\lambda_{j}+\lambda_{k}\right) u_{j}+\sum_{j=m+1}^{k-1}\left(\lambda_{j}-\lambda_{k}\right) u_{j}-v,
$$

so that $U^{\prime}=\left\{u_{k+1}, \ldots, u_{d+1}\right\}$ and $U^{\prime \prime}=\left\{u_{1}, \ldots, u_{k-1}\right\}$. Since we wish $U^{\prime}$ and $U^{\prime \prime}$ to contain $r$ vectors each, we are forced to have $k=r+1$.

We conclude that

$$
\begin{aligned}
G^{\prime} & =G_{r+2} \cap \cdots \cap G_{d+1}, \\
G^{\prime \prime} & =G_{1} \cap \cdots \cap G_{r} .
\end{aligned}
$$

Moreover, $G^{\prime} \cap G^{\prime \prime}$ is the unique vertex of the special face $G$ which does not lie in the facet $G_{r+1}$. Since $G^{\prime \prime} \subseteq G$, we see that $G^{\prime \prime}$ is of kind 0 and $G^{\prime}$ is thus of kind $m$ (alternatively, $\left\{u_{1}, \ldots, u_{m}\right\} \subseteq U^{\prime \prime}$, so that $U^{\prime}$ is missing of all of $\left\{u_{1}, \ldots, u_{m}\right\}$ ). It follows that the only contribution to $s^{2}$ comes from $G^{\prime}+G^{\prime \prime}$, and so has sign $(-1)^{m}$, which is the assertion of the lemma.

\section{References}

1. U. Betke. Mixed volumes of polytopes. Arch. Math. 58 (1992), 388-391.

2. L. J. Billera and C. W. Lee. A proof of the sufficiency of McMullen's conditions for $f$-vectors of simplicial convex polytopes. J. Combin. Theory Ser. A 31 (1981), 237-255.

3. L. J. Billera and B. Sturmfels. Fiber polytopes. Ann. of Math. 135 (1992), 527-549.

4. W. Fulton and B. Sturmfels. Intersection theory on toric varieties. Topology (to appear).

5. B. Grünbaum. Convex Polytopes. Wiley-Interscience, New York, 1967.

6. G. Kalai. Rigidity and the lower bound theorem, 1. Invent. Math. 88 (1987), 125-151.

7. C. W. Lee. Some recent results on convex polytopes. Contemp. Math. 114 (1990), 3-19.

8. C. W. Lee. Generalized stress and motions. In Polytopes: Abstract, Convex and Computational (ed. T. Bisztriczky, P. McMullen, R. Schneider, and A. I. Weiss). NATO ASI Series C, Vol. 440. Kluwer, Dordrecht, 1994, pp. 249-271.

9. C. W. Lee. P.1.-spheres, convex polytopes, and stress. Discrete Comput. Geom., this issue, pp. 389-421. 
10. P. McMullen. The numbers of faces of simplicial polytopes. Israel J. Math. 9 (1971), 559-570.

11. P. McMullen. The polytope algebra. Adv. in Math. 78 (1989), 76-130.

12. P. McMullen. On simple polytopes. Invent. Math. 113 (1993), 419-444.

13. P. McMullen. Separation in the polytope algebra. Beitr. Algebra Geom. 34 (1993), 15-30.

14. A. V. Pukhlikov and A. G. Khovanskir. Finitely additive measures of virtual polytopes. St. Petersburg Math. J. 4 (1993), 337-356 (transl. from Russian).

15. R. Schneider. Polytopes and Brunn-Minkowski theory. In Polytopes: Abstract, Convex and Computational (ed. T. Bisztriczky, P. McMullen, R. Schneider, and A. 1. Weiss). NATO ASI Series C, Vol. 440. Kluwer, Dordrecht, 1994, pp. 273-299.

16. R. P. Stanley. The numbers of faces of a simplicial convex polytope. Adv. in Math. 35 (1980), 236-238.

17. T.-S. Tay, N. White, and W. Whitely. Skeletal rigidity of simplicial complexes, 1, II. European J. Combin. 16 (1995), 381-403, 503-523.

18. D. W. Walkup and R. J.-B. Wets. Lifting projections of convex polyhedra. Pacific J. Math. 28 (1969), $465-475$.

Received October 5, 1994, and in revised form June 14, 1995. 